\title{
Comparação dos processos patológicos gerais no epitélio pulmonar de ratos espontaneamente hipertensos submetidos ao treinamento intervalado de alta
}

\section{intensidade}

\author{
Comparison of general pathological processes in the pulmonary epithelium of spontaneously \\ hypertensive rats submitted to high intensity interval training
}

Comparación de procesos patológicos generales en el epitelio pulmonar de ratas espontáneamente hipertensivas sometidas a entrenamiento en intervalos de alta intensidad

Recebido: 29/04/2021 | Revisado: 09/05/2021 | Aceito: 11/05/2021 | Publicado: 27/05/2021

\author{
Amanda Nogueira Soller Pires \\ ORCID: https://orcid.org/0000-0001-9934-5970 \\ Universidade do Oeste Paulista, Brasil \\ E-mail: mandahsoller@gmail.com \\ Fabiola de Azevedo Mello \\ ORCID: https://orcid.org/0000-0003-3373-0520 \\ Universidade do Oeste Paulista, Brasil \\ E-mail: Fabíola-azevedo@hotmail.com \\ Sérgio Marques Costa \\ ORCID: https://orcid.org/0000-0001-9287-065X \\ Universidade do Oeste Paulista, Brasil \\ E-mail: marxcosta@gmail.com \\ Daielli Chagas \\ ORCID: https://orcid.org/0000-0002-0908-5202 \\ Universidade do Oeste Paulista, Brasil \\ E-mail: daichagas76@gmail.com \\ Gabriela Vidotto Cavalieri \\ ORCID: https://orcid.org/0000-0002-8146-9614 \\ Universidade do Oeste Paulista, Brasil \\ E-mail: gabi_vidotto@hotmail.com \\ Ana Karina Marques Salge \\ ORCID: https://orcid.org/0000-0003-2718-1625 \\ Universidade Federal de Goiás, Brasil \\ E-mail: anasalge@gmail.com \\ Ana Paula Alves Favareto \\ ORCID: https://orcid.org/0000-0001-8634-9198 \\ Universidade do Oeste Paulista, Brasil \\ E-mail: anafavareto@unoeste.br \\ Debora Tavares de Resende e Silva \\ ORCID: https://orcid.org/0000-0002-3813-7139 \\ Universidade Federal da Fronteira Sul, Brasil \\ E-mail: debora.silva@uffs.edu.br \\ Francis Lopes Pacagnelli \\ ORCID: https://orcid.org/0000-0003-3712-5854 \\ Universidade do Oeste Paulista, Brasil \\ E-mail: francispacagnelli@unoeste.br \\ Renata Calciolari Rossi \\ ORCID: https://orcid.org/0000-0002-3311-064X \\ Universidade do Oeste Paulista, Brasil \\ E-mail: renatacalciolari@terra.com.br
}

\section{Resumo}

O objetivo foi comparar e quantificar morfologicamente o epitélio pulmonar de ratos hipertensos expostos ao treinamento de alta frequência intervalada (HIIT). Trata-se de um estudo experimental, no qual foram utilizados ratos Wistar Kyoto machos da linhagem espontaneamente hipertensos (SHR) (n=16) divididos em Grupo 01 (Espontaneamente hipertensos controles sedentário (SHR, $n=8)$ ) e Grupo 02 (Espontaneamente hipertensos treinados $(\mathrm{SHR}+\mathrm{T}, \mathrm{n}=8))$. O exercício foi realizado em esteira adaptada para roedores, por $50 \mathrm{minutos} / \mathrm{dia}, 5 \mathrm{dias} / \mathrm{semana}$ totalizando 8 semanas. Os intervalos entre tiros foram de 3 minutos com $60 \%$ da velocidade de exaustão. Após as 8 semanas de treinamento, os ratos foram submetidos a eutanásia e realização da autópsia para retirada dos pulmões. Os tecidos, após confecção das lâminas, foram corados com Hematoxilina e Eosina (HE) para avaliação do infiltrado 
inflamatório por meio da quantificação na região peribrônquica por método semiquantitativo. Foi observada uma redução significativa do peso dos ratos SHR+T quando comparado aos SHR sem intervenção. Na comparação morfométrica entre as áreas da via aérea, artéria pulmonar, luz da artéria pulmonar e à área alveolar não foram observadas diferenças estatisticamente significantes. Por meio do presente trabalho foi possível comprovar a eficácia do treinamento HIIT no condicionamento físico, perda de peso e com reflexo positivo no controle de hipertensão arterial, porém em relação à melhora do parênquima pulmonar não foi observado diferença nos resultados. Pensamos que se faz necessário um maior tempo de exercício para que haja tal mudança em alterações epiteliais e parênquima.

Palavras-chave: Treinamento intervalado de alta intensidade; Hipertensão; Ratos; Pulmão.

\begin{abstract}
The aim was to compare and morphologically quantify the pulmonary epithelium of hypertensive rats exposed to high intensity interval training (HIIT). This is an experimental study, in which male Wistar Kyoto rats of the spontaneously hypertensive strain (SHR) $(\mathrm{n}=16)$ were divided into Group 01 (Spontaneously hypertensive sedentary controls (SHR, $\mathrm{n}=8)$ ) and Group 02 (Spontaneously trained hypertensive patients (SHR $+\mathrm{T}, \mathrm{n}=8)$ ). The exercise was performed on a mat adapted for rodents, for 50 minutes / day, 5 days / week for a total of 8 weeks. The intervals between running shots were 3 minutes with $60 \%$ of the exhaustion speed. After 8 weeks of training, the rats were euthanized and performed an autopsy to remove the lungs. The tissues, after making the slides, were stained with Hematoxylin and Eosin (HE) to assess the inflammatory infiltrate by quantifying the peribronchial region using a semi-quantitative method. A significant reduction in the weight of SHR $+\mathrm{T}$ rats was observed when compared to SHR without intervention. In the morphometric comparison between the areas of the airway, pulmonary artery, pulmonary artery lumen and the alveolar area, no statistically significant differences were observed. Through this study it was possible to prove the effectiveness of HIIT training in physical conditioning, weight loss and with a positive impact on the control of arterial hypertension, however in relation to the improvement of the lung parenchyma observed a difference in the results. We think that a longer period of exercise is necessary for there to be such a change in epithelial and parenchymal changes.
\end{abstract}

Keywords: High intensity interval training; Hypertension; Rats; Lung.

\title{
Resumen
}

El objetivo fue comparar y cuantificar morfológicamente el epitelio pulmonar de ratas hipertensas expuestas a entrenamiento por intervalos de alta intensidad (HIIT). Se trata de un estudio experimental, en el que se dividieron ratas macho Wistar Kyoto de la cepa espontáneamente hipertensiva (SHR) $(\mathrm{n}=16)$ en el Grupo 01 (Controles sedentarios espontáneamente hipertensos (SHR, $\mathrm{n}=8)$ ) y el Grupo 02 (Hipertensivos entrenados espontáneamente pacientes $(\mathrm{SHR}+\mathrm{T}, \mathrm{n}=8)$ ). El ejercicio se realizó sobre una colchoneta adaptada para roedores, durante 50 minutos $/$ día, 5 días / semana durante un total de 8 semanas. Los intervalos entre tiros de carrera fueron de 3 minutos con el $60 \%$ de la velocidad de agotamiento. Después de 8 semanas de entrenamiento, las ratas fueron sacrificadas y se les realizó una autopsia para extirpar los pulmones. Los tejidos, después de realizar los portaobjetos, se tiñeron con Hematoxilina y Eosina (HE) para evaluar el infiltrado inflamatorio cuantificando la región peribronquial mediante un método semicuantitativo. Se observó una reducción significativa en el peso de las ratas SHR + T en comparación con SHR sin intervención. En la comparación morfométrica entre las áreas de la vía aérea, arteria pulmonar, luz de la arteria pulmonar y el área alveolar, no se observaron diferencias estadísticamente significativas. A través de este estudio se pudo comprobar la efectividad del entrenamiento HIIT en acondicionamiento físico, pérdida de peso y con un impacto positivo en el control de la hipertensión arterial, sin embargo, en relación con la mejora de la parénquima pulmonar, no se observó ninguna diferencia en los resultados. Creemos que es necesario un período más largo de ejercicio para que haya tal cambio en los cambios epiteliales y parenquimatosos.

Palabras clave: Entrenamiento por intervalos de alta intensidade; Hipertensión; Ratas; Pulmón.

\section{Introdução}

Segundo a Sociedade Brasileira de Cardiologia (2021), a Hipertensão Arterial Sistêmica (HAS) é uma condição clínica que se caracteriza pela elevação sustentada dos níveis pressóricos $\geq 140$ e/ou $90 \mathrm{mmHg}$. Frequentemente é associada a distúrbios metabólicos, alterações de órgãos-alvo em sua estrutura ou funcionamento, sendo agravada pela presença de outros fatores de risco (FR), como dislipidemia, obesidade, intolerância à glicose e diabetes melito (DM). Associa-se a eventos como morte súbita, acidente vascular cerebral (AVC), infarto agudo do miocárdio (IAM) e insuficiência cardíaca (IC) (Datasus; Issa et al., 2021).

A hipertensão pode se relacionar a alguns eventos de urgência hipertensiva (UH) e emergência hipertensiva (EH), que se não tratadas prontamente, podem culminar em eventos com maior morbidade (Ferreira et al., 2017). O tratamento da hipertensão consiste no uso de uma vasta gama de medicações, desde diuréticos (BLOWEY, 2016), vasodilatadores diretos 
(Elmi-Sarabi et al., 2017), inibidores adrenérgicos e beta bloqueadores (Garcia-Lunar et al., 2020), e inibidores da enzima convertedora de angiotensinogenio (IECA) (Mullick et al., 2017).

Essas medicações possuem diferentes mecanismos de ação e sua indicação é individualizada. Em paralelo ao uso destas medicações quando indicadas, a primeira fase do tratamento consiste em mudança de estilo de vida (MEV), compreendendo dieta e exercícios físicos de forma responsável e regular. A grande barreira encontrada na maioria dos relatos é a baixa adesão as medidas não farmacológicas e a MEV (Mills et al., 2020).

$\mathrm{O}$ método de treinamento intervalado de de alta intensidade (high intensity interval training - HIIT) vem chamando a atenção por ser um método de treinamento que alterna períodos de exercício aeróbio em alta intensidade com períodos de recuperação passiva ou ativa em uma intensidade moderada-baixa sendo usualmente praticado em bicicletas ou em esteiras ergométricas (Hannan et al., 2018). O HIIT tem como uma de suas vantagens o fato de ser de curta duração, o que seria altamente vantajoso na sociedade moderna, pricipalmente pela rotina sobrecarregada (Dalpiaz et al., 2016; Costa et al., 2018).

São abordados nove variáveis para a elaboração de protocolos de HIIT. Destas, mostraram se mais importantes: intensidade e a duração da relação trabalho-recuperação, seguidas do número de repetições, número de séries e da duração do período de recuperação entre as séries. Ainda, a modalidade de exercício deve ser considerada como fator de peso na elaboração das sessões de exercícios. A prescrição de um protocolo de HIIT é individualizado, variando com o estímulo e a adaptação desejados, tornando-o um instrumento versátil na elaboração de um protocolo de reabilitação cardíaca (Buchheit et al., 2013; Vilela-Martin et al., 2020).

Para a prescrição do treinamento HIIT na população hipertensa, afigura-se um melhor esclarecimento acerca da influência do HIIT na pressão arterial (PA) e nos órgãos afetados, por ser um método facilmente implantável, de baixo custo e com grande potencial de uso.

Ao contrário do que se imagina, a HAS não está relacionada apenas ao sistema cardiovascular, existem estudos que demonstram que alterações pulmonares como Edema Agudo de Pulmão (EAP) causados pela HAS, e portanto, o EAP é considerado uma emergências hipertensivas (Malachias et al., 2016). A relação pulmonar com a HAS também está dentro das investigações diagnósticas dos hipertensos, visto que na presença de suspeita de insuficiência cardíaca nesses pacientes, é necessário investigar se há comprometimento pulmonar que possa estar influenciando essa insuficiência, principalmente para descartar Hipertensão Arterial Pulmonar, que é considerada uma doença grave (Yamada et al., 2011).

Pesquisadores demonstraram que o HIIT realizado por adultos jovens do sexo masculino no período de duas semanas foi capaz de melhorar a função mitocondrial do músculo esquelético e também a cinética de consumo de oxigênio pulmonar, ou seja, evidenciou o benefício desse exercício tanto para a saúde cardiovascularar, quanto para a saúde respiratória (Christensen et al., 2016). Entretanto, não foram encontrados na literatura trabalhos que avaliassem o efeito do exercício no epitélio pulmonar.

Sendo assim, a importância da avaliação do tecido pulmonar em hipertensos e a análise da influência dos exercícios físicos relaciona-se ao fato de trazer subsídios aos profissionais de saúde atuantes com hipertensos, a fim de elencar se há possibilidade de melhora do tecido pulmonar e pressão arterial por meio do HIIT. Grande parte dos hipertensos não tem ciência dos riscos a que estão expostos. Em decorrência deste fato, novas técnicas e tratamentos tem como objetivo diminuir a pressão arterial por meio de métodos mais simples e fáceis de serem aderidos. O HIIT, quando bem prescrito e indicado, é uma técnica que consome pouco tempo, o que seria uma vantagem sobre outros métodos de exercício. Desta forma, o objetivo deste estudo foi comparar e quantificar morfologicamente o epitélio pulmonar de ratos hipertensos expostos ao HIIT. 


\section{Metodologia}

Foram utilizados ratos Wistar Kyoto machos da linhagem espontaneamente hipertensos (SHR) (n=16) com quatro meses de idade. Os animais pesavam entre 250 - 300 gramas, provenientes do Biotério Central da Universidade Estadual de Campinas - UNICAMP. Foram mantidos no Laboratório de Experimentação Animal da Universidade Júlio de Mesquita Filho - UNESP, Campus Botucatu - São Paulo e divididos em dois grupos:

Grupos 01 - Espontaneamente hipertensos controle sedentário (SHR, n=8).

Grupo 02 - Espontaneamente hipertensos treinados (SHR+T, n=8), Dalpiaz et al., 2016.

Foram alojados em caixas plásticas (dimensão 41 x 34 x $16 \mathrm{~cm}$ ) contendo de 3 a 5 animais em cada. A temperatura foi controlada entre $21 \mathrm{o}$ e $23 \mathrm{o} \mathrm{C}$ e umidade relativa do ar de $50 \%$ a $60 \%$, com ciclos de luminosidade de $12 \mathrm{~h}$ (claro-escuro) com início do ciclo claro as 7h. Os ratos receberam ração (Supralab, Alisul R, Brasil) e água ad libitum. Os procedimentos realizados e utilizados foram aprovados pelo Comitê de Ética em Pesquisa da Universidade do Oeste Paulista, Presidente Prudente $\left(\mathrm{N}^{\circ} 4923\right)$.

O exercício foi realizado em esteira adaptada para roedores (modelo TK 1, IMBRAMED), por 50 minutos/dia, 5 dias/semana totalizando 8 semanas. $\mathrm{O}$ treino foi realizado das $14 \mathrm{~h} 00 \mathrm{~min}$ às $14 \mathrm{~h} 50 \mathrm{~min}$. Inicialmente, os animais foram submetidos a uma adaptação para o treinamento HIIT, que foi uma corrida em esteira na velocidade de $6 \mathrm{~m} / \mathrm{mim}$, por cinco minutos, no decorrer dos cinco dias antes do treinamento. Após adaptação, foram submetidos a um teste para avaliar a velocidade máxima de exaustão e coleta do lactato. Ao início dos testes, os ratos foram colocados na esteira durante 5 dias, sendo $10 \mathrm{~min} / \mathrm{dia}$. Neste momento, sem inclinação e iniciado com uma velocidade de $6 \mathrm{~m} / \mathrm{min}$, aumentando $3 \mathrm{~m} / \mathrm{min}$ a cada 3 minutos, até que os ratos fossem incapazes de correr (de Vargas et al., 2017). Os animais considerados exaustos eram incapazes de continuarem o exercício por incoordenação motora ou pouca reatividade após estímulos.

As sessões planejadas tinham protocoladas três fases: a) aquecimento, b) HIIT c) desaquecimento. Sendo o aquecimento com 5 minutos a $60 \%$ da velocidade máxima de exaustão, o HIIT com 100\% da velocidade atingida no teste de exaustão $(21 \mathrm{~m} / \mathrm{mim})$ por 4 minutos, seguidos por $60 \%$ da velocidade de exaustão por 3 minutos repetidos por cinco vezes durante 1 semana (Haram et al, 2009). Nas semanas seguintes (segunda, terceira e quarta semanas), o HIIT foi realizado mantendo a mesma velocidade da primeira semana, porém, repetidos por 6 e 7 vezes, consecutivamente.

Antes de iniciar a quarta semana foi realizado um segundo teste para a avaliação da velocidade máxima de exaustão. $\mathrm{Na}$ quinta e na sexta semanas, os ratos correram com $100 \%$ da velocidade atingida no teste de exaustão $(27 \mathrm{~m} / \mathrm{min})$ por 4 minutos, seguidos por 3 minutos a $60 \%$ da velocidade de exaustão $(16 \mathrm{~m} / \mathrm{min})$, repetidos por 7 vezes durante 1 semana. Na sétima semana houve um aumento diário de $3 \%$ na velocidade ( $15 \%$ semanal- $28,29,30 \mathrm{~mm} / \mathrm{min}$ ) por 4 minutos, seguidos por 3 minutos a $60 \%$ da velocidade de exaustão $(16,17$ e $18 \mathrm{~mm} / \mathrm{min})$, repetidos por sete vezes. Na oitava semana ocorreu um aumento de $18 \%$ na velocidade ( $32 \mathrm{~m} / \mathrm{min}$ ) por 4 minutos, seguidos por 3 minutos a $60 \%$ da velocidade de exaustão $(19 \mathrm{~m} / \mathrm{min})$ repetidos por sete vezes. O período de desaquecimento foi realizado por 5 minutos a $60 \%$ da velocidade de exaustão.

Os ratos foram submetidos a eutanásia e realização da autópsia para retirada dos pulmões. Para que os pulmões pudessem manter sua arquitetura morfológica e pressão constante $(20 \mathrm{cmH} 2 \mathrm{O})$, foi introduzida formalina tamponada $10 \%$ na região da traqueia. O tecido pulmonar foi coletado, aleatoriamente, de áreas centrais e periféricas dos pulmões de cada animal para o processamento e confecção dos blocos em parafina. Foram realizados cortes de $5 \mu \mathrm{m}$ nas amostras corados com Hematoxilina e Eosina (HE) para que fosse possível a seleção das vias aéreas mais apropriadas para avaliação do infiltrado inflamatório.

Após a coloração, as células foram quantificadas na região peribrônquica onde procedeu-se a avaliação do infiltrado inflamatório por método semiquantitativo proposto por Carnielli (2010).

Este sistema classifica o infiltrado inflamatório em escores de 1 a 3 , sendo: 
1. (áreas de inflamação ocasionais);

2. (inflamação em maioria das áreas, cercadas por uma camada fina de células)

3. (maioria das áreas inflamadas circundadas por uma camada grossa de células).

Analisou-se também as lâminas com as vias aéreas localizadas em região distal do pulmão e com preservação da arquitetura histológica. Para a investigação da secreção de muco, foram utilizadas preparações com a coloração de Alcian Blue. As lâminas contendo os cortes histológicos foram cegadas para que os examinadores não soubessem o grupo analisado e, desta forma, emitissem falsos resultados.

Vias aéreas: Coletou-se duas amostras de vias aéreas para a realização da morfometria. De cada via aérea, foram coletadas medidas referentes a área da muscular da via aérea e área da luz da via aérea. Os valores então foram normalizados pela relação entre área da via aérea/área da luz da via aérea.

Artéria pulmonar: Foram coletados dois ramos de artéria pulmonar para a realização da morfometria. De cada ramo de artéria, coletaram-se medidas referentes a área de músculo liso da artéria pulmonar e área da luz da artéria pulmonar. Posteriormente, os valores foram normalizados pela relação entre área da muscular/área da luz do vaso.

Análise estatística: Foi utilizado o programa Sigma Stat ${ }^{\circ}$ versão 2.0. Os casos em que os dados apresentaram as distribuições normais e homocedásticas, foram analisados utilizando-se de testes paramétricos como a análise de variância (ANOVA) seguidos pelo teste de Tukey. Utilizou-se também o teste não paramétrico de Kruskal-Wallis. As diferenças foram consideradas estatisticamente significantes quando $\mathrm{p}$ foi menor que $5 \%(\mathrm{p}<0,05)$.

\section{Resultados}

Na comparação entre o peso dos animais experimentais, foi observada uma redução significativa do peso dos ratos SHR+HITT quando comparado aos SHR sem intervenção ( $p=0,002)$ (Figura 1).

Figura 1. Comparação do peso dos ratos espontaneamente hipertensos submetidos ao treinamento intervalado de alta intensidade.

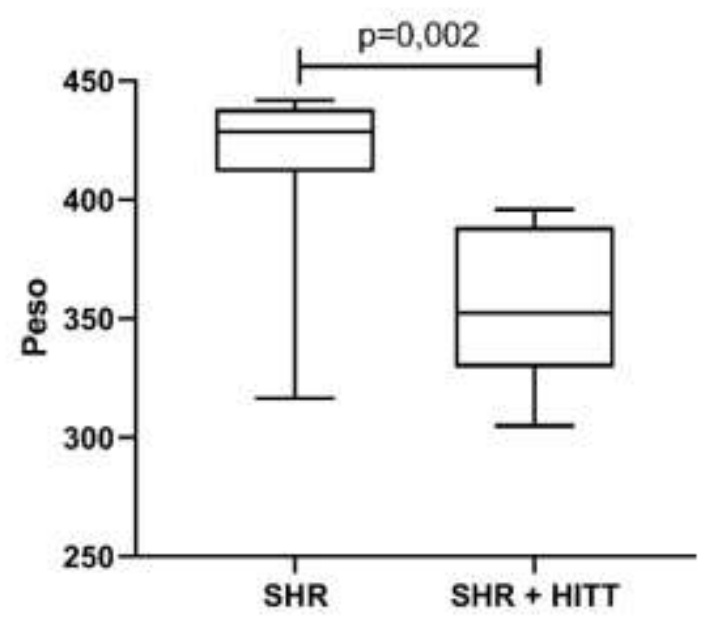

Fonte: Autores.

Na comparação morfométrica entre as áreas da via aérea, artéria pulmonar e luz da artéria pulmonar, não foiobservado resultados estatisticamente significativos (Tabela 1). 
Tabela 1. Comparação morfométrica das vias aéreas e artéria pulmonar de ratos SHR submetidos ao HITT.

\begin{tabular}{lccc}
\hline & 1. SHR & 2. SHR + HIIT & Valor de p \\
\hline Via aérea & $2,39(1,45-4,49)$ & $2,23(1,91-5,82)$ & 0,703 \\
Vaso & $1,59(1,30-5,75)$ & $1,72(1,32-3,88)$ & 0,849 \\
Luz do vaso & $3,50(2,82-7,56)$ & $1,50(1,21-4,61)$ & 0.095 \\
\hline
\end{tabular}

Fonte: Autores.

Em relação á área alveolar, também não foram verificadas diferenças significativas quando comparados os grupos SHR com SHR+ HITT (Figuras 1 e 2).

Figura 2. Área alveolar dos animais SHR e SHR + HITT.

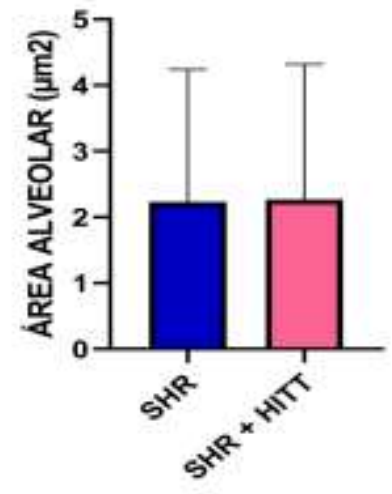

Fonte: Autores.

\section{Discussão}

Atualmente percebemos que a preocupação da população a respeito de bons hábitos de higiene e saúde vem aumentando muito. Os indivíduos encontram-se mais conscientes do impacto que os bons hábitos de vida, boa alimentação e exercícios físicos tem sobre a qualidade de vida (Malta et al, 2014; Laverack, 2017).

Arquivos do Ministério da Saúde (BVMS) demonstram uma vasta gama de estudos voltados para a hipertensão e suas repercussões na sociedade. Quando confrontamos o HIIT com esses dados, percebemos que ele se trata de uma excelente ferramenta para combate do sedentarismo e hipertensão e, como consequência, conseguimos atingir doenças que são decorrentes dos quadros de hipertensão sustentada (AVC, Infarto, Arterioescleroses) (Datasus).

Ao iniciar as provas com os animais deste estudo, foi preciso realizar o condicionamento físico e a prova de exaustão dos animais. Neste momento, os ratos foram expostos a treinos progressivos em esteira adaptada para roedores e, desta forma, percebemos que os animais apresentaram melhora em seu condicionamento físico, confirmando o fato do HIIT ser uma boa ferramenta para o combate do sedentarismo, como citado anteriormente.

Além disso, estudos anteriores demonstraram resultados semelhantes em relação a perda de peso e melhora do condicionamento e resistência após a realização do HIIT em ratos Zucker (Maillard et al., 2019), crianças obesas (Liu, et al., 2019), mulheres com sobrepeso (Mirghani et al., 2019), e homens obesos (Smith-Ryan et al., 2015) o qual evidencia o benefício desse exercício, principalmente pelo fato de que a maioria das pessoas que praticam o HIIT, buscam pelo emagrecimento e melhora do condicionamento físico (Ito, 2019; Guio et al., 2019).

Não foram encontrados na literatura estudos sobre o efeito do HIIT diretamente no epitélio pulmonar, porém, estudos 
compararam o efeito do HIIT com exercícios contínuo na capacidade funcional e variáveis cardiovasculares em pacientes com Doença Pulmonar Obstrutiva Crônica (DPOC) por meio de uma meta-análise e evidenciaram que tanto o HIIT quanto o exercício contínuo atuam de forma semelhante em relação às variáveis analisadas (consumo máximo de oxigênio absoluto e relativo, função endotelial, pressão arterial sistólica e diastólica) (Adolfo et al., 2019).

Ainda sobre pacientes com DPOC, o American College of Sports Medicine recomenda o exercício aeróbio contínuo de intensidade moderada, de 20 a 60 min de duração por sessão, visto que já foi demonstrado que apresenta benefícios fisiológicos (Spruit et al., 2013). E além desse exercício, outros autores recomendam também o HIIT como uma alternativa aos treinamentos com exercícios contínuos para indivíduos com DPOC que possuem dificuldade em conseguir atingir a duração alvo em razão de dispneia, fadiga ou outro sintoma (Camilo et al., 2011).

Como observado anteriormente, os estudos com seres humanos demonstraram benefícios a saúde pulmonar e cardiovascular, tanto com o HIIT, quanto com exercícios convencionais (aeróbicos e contínuos), entretanto ainda não foi demonstrado o mecanismo que os exercícios desencadeiam no tecido pulmonar, sendo esse o nosso objetivo no presente trabalho.

\section{Conclusão}

Diante do término do estudo, não encontramos valores com adequada significância e, pode-se sugerir que o tempo que foi adotado no estudo não permitiu que o parênquima pulmonar, bem como os demais tecidos estudados, passassem pelo processo de metaplasia. Mesmo que a mudança de peso se evidenciou, esta ocorre muito mais precocemente quando comparada com a mudança epitelial. Sendo assim, os tecidos precisam de mais tempo para que se manifestem modificações em sua conformação e qualidade.

Por meio do presente trabalho foi possível comprovar a eficácia do treinamento HIIT no condicionamento físico, perda de peso e com reflexo positivo no controle de hipertensão arterial, com consequente melhora da qualidade de vida.

\section{Referências}

Adolfo, J. R., Dhein, W., \& Sbruzzi, G. (2019). Diferentes intensidades de exercício físico e capacidade funcional na DPOC: revisão sistemática e metaanálise. Jornal Brasileiro de Pneumologia, 45(6).

Brasil. Ministério da Saúde. Departamento de Informática do Sistema Único de Saúde (DATASUS) [online]. Brasília [s.d.]. http:// www.datasus.gov.br. Acessado em 25 de março de 2021.

Blowey, D. L. (2016). Diuretics in the treatment of hypertension. Pediatric nephrology, 31(12), 2223-2233.

Buchheit, M., \& Laursen, P. B. (2013). High-intensity interval training, solutions to the programming puzzle. Sports medicine, 43(5), $313-338$.

Camillo, C. A., de Moraes Laburu, V., Gonçalves, N. S., Cavalheri, V., Tomasi, F. P., Hernandes, N. A., ... \& Pitta, F. (2011). Improvement of heart rate variability after exercise training and its predictors in COPD. Respiratory medicine, 105(7), 1054-1062.

Carnieli, D. S. (2010). Inflamação e remodelamento pulmonar em camundongos com sensibilização alérgica em diferentes idades: um estudo comparativo (Doctoral dissertation, Universidade de São Paulo).

Christensen, P. M., Jacobs, R. A., Bonne, T., Flück, D., Bangsbo, J., \& Lundby, C. (2016). A short period of high-intensity interval training improves skeletal muscle mitochondrial function and pulmonary oxygen uptake kinetics. Journal of Applied Physiology, 120(11), 1319-1327.

Costa, E. C., Hay, J. L., Kehler, D. S., Boreskie, K. F., Arora, R. C., Umpierre, D., ... \& Duhamel, T. A. (2018). Effects of high-intensity interval training versus moderate-intensity continuous training on blood pressure in adults with pre-to established hypertension: a systematic review and meta-analysis of randomized trials. Sports Medicine, 48(9), 2127-2142.

Dalpiaz, M. R., Dorneles, G. P., Souza, M. P., Peres, A., Monteiro, M. B., \& Souza, M. P. (2016). Treinamento Intervalado de Alta Intensidade: quebrando paradigmas na reabilitação cardiovascular. Revista Brasileira de Prescrição e Fisiologia do Exercício (RBPFEX), 10(57), 16-28.

de Vargas, L. D. S., das Neves, B. H. S., Roehrs, R., Izquierdo, I., \& Mello-Carpes, P. (2017). One-single physical exercise session after object recognition learning promotes memory persistence through hippocampal noradrenergic mechanisms. Behavioural brain research, 329, $120-126$.

Elmi-Sarabi, M., Deschamps, A., Delisle, S., Ased, H., Haddad, F., Lamarche, Y., ... \& Denault, A. Y. (2017). Aerosolized vasodilators for the treatment of pulmonary hypertension in cardiac surgical patients: a systematic review and meta-analysis. Anesthesia \& Analgesia, 125(2), 393-402. 
Ferreira, N. Z., de Souza, F. L. A., Mariano, T. B., Carrara, B., Collegio, G., Molinari, A. O., ... \& Pacagnelli, F. L. (2017). efeito hipotensor do exercício intervalado de alta intensidade em animais espontaneamente hipertensos. In Colloquium Vitae. ISSN: 1984-6436 (Vol. 9, No. 3, pp. 31-35).

Garcia-Lunar, I., Blanco, I., Fernández-Friera, L., Prat-Gonzàlez, S., Jordà, P., Sánchez, J., ... \& García-Álvarez, A. (2020). Design of the ß3-adrenergic agonist treatment in chronic pulmonary hypertension secondary to heart failure trial. Basic to Translational Science, 5(4), 317-327.

Guio de Prada, V., Ortega, J. F., Morales-Palomo, F., Ramirez-Jimenez, M., Moreno-Cabañas, A., \& Mora-Rodriguez, R. (2019). Women with metabolic syndrome show similar health benefits from high-intensity interval training than men. PloS One, 14(12), e0225893.

Hannan, A. L., Hing, W., Simas, V., Climstein, M., Coombes, J. S., Jayasinghe, R., ... \& Furness, J. (2018). High-intensity interval training versus moderateintensity continuous training within cardiac rehabilitation: a systematic review and meta-analysis. Open access journal of sports medicine, 9 , 1.

Haram, P. M., Kemi, O. J., Lee, S. J., Bendheim, M. Ø., Al-Share, Q. Y., Waldum, H. L., ... \& Wisløff, U. (2009). Aerobic interval training vs. continuous moderate exercise in the metabolic syndrome of rats artificially selected for low aerobic capacity. Cardiovascular research, 81(4), 723-732.

Issa, A. F. C., Nascimento, B. R., Correa Filho, H., Vieira, M. L. C., Barroso, W. K. S., Rodrigues, C. S., ... \& Mota-Gomes, M. A. (2021). Diretrizes Brasileiras de Hipertensão Arterial-2020. Arq Bras Cardiol, 116(3), 516-658.

Ito, S. (2019). High-intensity interval training for health benefits and care of cardiac diseases-the key to an efficient exercise protocol. World Journal of Cardiology, 11(7), 171 .

Laverack, G. (2017). The challenge of behaviour change and health promotion. Challenges, 8(2), 25.

Liu, J. X., Zhu, L., \& Deng, J. M. (2019). The effects of high-intensity interval training versus moderate-intensity continuous training on fat loss and cardiometabolic health in pediatric obesity: A protocol of systematic review and meta-analysis. Medicine, 98(10).

Malachias, M. V. B., Póvoa, R. M. S., Nogueira, A. R., Souza, D., Costa, L. S., \& Magalhães, M. E. (2016). $7^{a}$ Diretriz Brasileira de Hipertensão Arterial: Capítulo 3-Avaliação Clínica e Complementar. Arquivos Brasileiros de Cardiologia, 107(3), 14-17.

Maillard, F., Vazeille, E., Sauvanet, P., Sirvent, P., Combaret, L., Sourdrille, A., ... \& Boisseau, N. (2019). High intensity interval training promotes total and visceral fat mass loss in obese Zucker rats without modulating gut microbiota. PLoS One, 14(4), e0214660.

Malta, D. C., Moura, L. D., Prado, R. R. D., Escalante, J. C., Schmidt, M. I., \& Duncan, B. B. (2014). Mortalidade por doenças crônicas não transmissíveis no Brasil e suas regiões, 2000 a 2011. Epidemiologia e Serviços de Saúde, 23, 599-608.

Mills, K. T., Stefanescu, A., \& He, J. (2020). The global epidemiology of hypertension. Nature Reviews Nephrology, 16(4), 223-237.

Mirghani, S. J., Seydyousefi, M., Pekkala, S., Sharifian, S., \& Beyshami, G. (2019). Shorter recovery time following high-intensity interval training induced higher body fat loss among overweight women. Sport Sciences for Health, 15(1), 157-165.

Mullick, A. E., Yeh, S. T., Graham, M. J., Engelhardt, J. A., Prakash, T. P., \& Crooke, R. M. (2017). Blood pressure lowering and safety improvements with liver angiotensinogen inhibition in models of hypertension and kidney injury. Hypertension, 70(3), 566-576.

Smith-Ryan, A. E., Melvin, M. N., \& Wingfield, H. L. (2015). High-intensity interval training: Modulating interval duration in overweight/obese men. The Physician and sportsmedicine, 43(2), 107-113.

Spruit, M. A., Singh, S. J., Garvey, C., ZuWallack, R., Nici, L., Rochester, C., ... \& Wouters, E. F. (2013). An official American Thoracic Society/European Respiratory Society statement: key concepts and advances in pulmonary rehabilitation. American journal of respiratory and critical care medicine, 188(8), e13-e64.

Vilela-Martin, J. F., Yugar-Toledo, J. C., Rodrigues, M. D. C., Barroso, W. K. S., Carvalho, L. C. B. S., González, F. J. T., ... \& Póvoa, R. M. D. S. (2020). Posicionamento Luso-Brasileiro de Emergências Hipertensivas-2020. Arquivos Brasileiros de Cardiologia, 114(4), 736-751.

Yamada, A. T. T., Lavras, C., \& DEMUNER, M. (2011). Manual de orientação clínica: hipertensão arterial sistêmica (HAS). São Paulo: SES. 